\title{
The use of two-point Taylor expansions in singular one-dimensional boundary value problems I
}

\author{
Chelo Ferreira ${ }^{1}$, José L. López ${ }^{2}$ and Ester Pérez Sinusía ${ }^{1}$ \\ ${ }^{1}$ Dpto. de Matemática Aplicada, IUMA, Universidad de Zaragoza \\ e-mail: cferrei@unizar.es, ester.perez@unizar.es \\ ${ }^{2}$ Dpto. de Ingeniería Matemática e Informática, Universidad Pública de Navarra and INAMAT, Navarra \\ e-mail: jl.lopez@unavarra.es
}

\begin{abstract}
We consider the second-order linear differential equation $(x+1) y^{\prime \prime}+f(x) y^{\prime}+g(x) y=h(x)$ in the interval $(-1,1)$ with initial conditions or boundary conditions (Dirichlet, Neumann or mixed Dirichlet-Neumann). The functions $f(x), g(x)$ and $h(x)$ are analytic in a Cassini disk $\mathcal{D}_{r}$ with foci at $x= \pm 1$ containing the interval $[-1,1]$. Then, the end point of the interval $x=-1$ may be a regular singular point of the differential equation. The two-point Taylor expansion of the solution $y(x)$ at the end points \pm 1 is used to study the space of analytic solutions in $\mathcal{D}_{r}$ of the differential equation, and to give a criterion for the existence and uniqueness of analytic solutions of the boundary value problem. This method is constructive and provides the two-point Taylor approximation of the analytic solutions when they exist.
\end{abstract}

2010 AMS Mathematics Subject Classification: 34A25, 34B05, 41A58.

Keywords \& Phrases: Second-order linear differential equations, regular singular point, boundary value problem, Frobenius method, two-point Taylor expansions.

\section{Introduction}

In [8] we considered the second-order linear equation $y^{\prime \prime}+f(x) y^{\prime}+g(x) y=h(x)$ in the interval $(-1,1)$ with initial conditions or boundary conditions of the type Dirichlet, Neumann or mixed DirichletNeumann. The functions $f(x), g(x)$ and $h(x)$ are analytic in a Cassini disk with foci at $x= \pm 1$ containing the interval $[-1,1]$. Then, the end points of the interval, where the boundary data are given, are regular points of the differential equation. The two-point Taylor expansion of the solution $y(x)$ at the end points \pm 1 was used to give a criterion for the existence and uniqueness of analytic solutions of the initial or boundary value problem and approximate the solutions when they exist.

In this paper we continue our investigation considering problems that have an extra difficulty: one of the end points of the interval is a regular singular point of the differential equation. These problems are of great interest, since many special functions of the mathematical physics satisfy this type of equations [10]. We consider initial or boundary value problems of the form:

$$
\left\{\begin{array}{l}
(x+1) y^{\prime \prime}+f(x) y^{\prime}+g(x) y=h(x) \text { in }(-1,1), \\
B\left(\begin{array}{c}
y(-1) \\
y(1) \\
y^{\prime}(-1) \\
y^{\prime}(1)
\end{array}\right)=\left(\begin{array}{l}
\alpha \\
\beta
\end{array}\right),
\end{array}\right.
$$


where $f, g$ and $h$ are analytic in a Cassini disk with foci at $x= \pm 1$ containing the interval $[-1,1]$ (we give more details in the next section), $\alpha, \beta \in \mathbb{C}$ and $B$ is a $2 \times 4$ matrix of rank two which defines the initial conditions or the boundary conditions (Dirichlet, Neumann or mixed).

The consideration of the interval $(-1,1)$ is not a restriction, as any real interval $(a, b)$ can be transformed into the interval $(-1,1)$ by means of an affine change of the independent variable. The form of the differential equation in (1) is not a restriction either: consider a differential equation of the form $(x+1)^{2} u^{\prime \prime}(x)+(x+1) F(x) u^{\prime}(x)+G(x) u(x)=0$, with $F$ and $G$ analytic at $x=-1$. After the change of the dependent variable $u=(x+1)^{\lambda} y$, with $\lambda$ a solution of the equation $\lambda(\lambda-1)+$ $F(-1) \lambda+G(-1)=0$, the equation may be written in the form $(x+1) y^{\prime \prime}+f(x) y^{\prime}+g(x) y=0$, with $f$ and $g$ analytic at $x=-1$. On the other hand, the point $x=-1$ is indeed a regular singular point of the differential equation when $|f(-1)|+|g(-1)|+|h(-1)| \neq 0$; if $f(-1)=g(-1)=h(-1)=0$, then $x=-1$ is a regular point, and problem (1) is the regular problem analyzed in [8]. We omit this restriction here and then, the regular case studied in [8] may be considered a particular case of the more general one analyzed in this paper.

A standard theorem for the existence and uniqueness of solution of (1) is based on the knowledge of the two-dimensional linear space of solutions of the homogeneous equation $(x+1) y^{\prime \prime}+f(x) y^{\prime}+g(x) y=0$ [3, Chap. 4, Sec. 1]. When $f$ are $g$ are constants or in some other particular situation, it is possible to find the general solution of the equation (sometimes via the Green function [3, Chap. 4], [13, Chaps. 1 and 3])). But this is not possible in general, and that standard criterion for the existence and uniqueness of solution of (1) is not practical. Other well-known criterion for the existence and uniqueness of solution of (1) is based on the Lax-Milgram theorem when (1) is an elliptic problem [4]. In any case, the determination of the existence and uniqueness of solution of (1) requires a non-systematic detailed study of the problem, like for example the study of the eigenvalue problem associated to (1) [3, Chap. 4, Sec. 2], [13, Chap. 7].

When $f, g$ and $h$ are analytic in a disk with center at $x=0$ and containing the interval $[-1,1]$, we may consider the initial value problem:

$$
\left\{\begin{array}{l}
(x+1) y^{\prime \prime}+f(x) y^{\prime}+g(x) y=h(x), \quad x \in(-1,1), \\
y(0)=y_{0}, \quad y^{\prime}(0)=y_{0}^{\prime},
\end{array}\right.
$$

with $y_{0}, y_{0}^{\prime} \in \mathbb{C}$. Using the Frobenius method we can approximate the solution of this problem by its Taylor polynomial of degree $N \in \mathbb{N}$ at $x=0, y_{N}(x)=\sum_{n=0}^{N} c_{k} x^{k}$, where the coefficients $c_{k}$ are affine functions of $c_{0}=y_{0}$ and $c_{1}=y_{0}^{\prime}$. By imposing the boundary conditions given in (1) over $y_{N}(x)$, we obtain an algebraic linear system for $y_{0}$ and $y_{0}^{\prime}$. The existence and uniqueness of solution of this algebraic linear system gives us information about the existence and uniqueness of solution of (1). This procedure, although theoretically possible, has a difficult practical implementation since the data of the problem are given at $x= \pm 1$, not at $x=0$ (see $[2,12]$ ). Moreover, when $f, g$ or $h$ have a singularity close to the interval $[-1,1]$, the above mentioned disk does not contain the interval $[-1,1]$ and the Taylor series of the solution $y(x)$ does not converge $\forall x \in[-1,1]$. In this case we can use a Taylor expansion of the solution at several points along the interval $[-1,1]$ and match these expansions at intersecting disks [9, Sec. 7]. In this way, we obtain an approximation of the solution of (1) in the form of a piecewise polynomial in several subintervals of $[-1,1]$. But this approximation is not uniform in the whole interval $[-1,1]$ and the matching of the expansions translates into numerical errors.

In [8] we improved the ideas of the previous paragraph for the regular case (when $f(-1)=g(-1)=$ $h(-1)=0$ ) using, not the standard Taylor expansion in the associated initial value problem (2), but a two-point Taylor expansion [6] at the end points $x= \pm 1$ directly in the differential equation and in the boundary conditions. The convergence region for a two-point Taylor expansion is a Cassini disk (see Fig. 1 below), and this Cassini disk avoids the possible singularities of the coefficient functions located near the interval $[-1,1]$ more efficiently than the standard Taylor disk [7].

In this paper we investigate if a two-point Taylor expansion at the end points $x= \pm 1$ also works for the more general problem (1), in particular when $x=-1$ is a regular singular point of the equation. 
Thus, we use the two-point Taylor expansion of the solution $y(x)$ to give a criterion for the existence and uniqueness of analytic solutions based on the data of the problem, not based on the knowledge of the general solution of the differential equation.

The paper is organized as follows. In the next section we introduce some elements of the theory of two-point Taylor expansions and study the space $S$ of analytic solutions of the differential equation $(x+1) y^{\prime \prime}+f(x) y^{\prime}+g(x) y=h(x)$. In Section 3 we derive the two-point Taylor expansion at the end points $x= \pm 1$ of the functions of $S$ (when $S$ is nonempty). In Section 4 we give an algebraic characterization of $S$ that we use, in Section 5, to formulate a criterion of existence and uniqueness of analytic solutions of problem (1). In Section 6 we give some illustrative examples. Section 7 contains some final remarks.

\section{Global analytic solutions of the differential equation}

Assume that the coefficient functions $f, g$ and $h$ in (1) are analytic in the Cassini disk $\mathcal{D}_{r}=\{z \in \mathbb{C} \mid$ $\left.\left|z^{2}-1\right|<r\right\}$ with foci at $z= \pm 1$ and Cassini's radius $r$, with $r>1$ (see [6]). The requirement $r>1$ assures that the interval $[-1,1]$ is contained into the Cassini disk $\mathcal{D}_{r}$ (see Fig. 1). Then, the three functions $f, g$ and $h$, admit a two-point Taylor series in $\mathcal{D}_{r}$ of the form [6],

$$
f(z)=\sum_{n=0}^{\infty}\left[f_{n}^{0}+f_{n}^{1} z\right]\left(z^{2}-1\right)^{n}, \quad g(z)=\sum_{n=0}^{\infty}\left[g_{n}^{0}+g_{n}^{1} z\right]\left(z^{2}-1\right)^{n}, \quad h(z)=\sum_{n=0}^{\infty}\left[h_{n}^{0}+h_{n}^{1} z\right]\left(z^{2}-1\right)^{n},
$$

where the coefficients of the expansions of $f(z)$ are $[6]$

$$
\begin{aligned}
f_{0}^{0} & :=\frac{f(1)+f(-1)}{2}, \quad f_{0}^{1}:=\frac{f(1)-f(-1)}{2}, \\
f_{n}^{0} & :=\sum_{k=0}^{n} \frac{(n+k-1) !}{(n-k-1) !} \frac{(-1)^{k} f^{(n-k)}(1)+(-1)^{n} f^{(n-k)}(-1)}{n ! k ! 2^{n+k+1}}, \quad n=1,2,3, \ldots, \\
f_{n}^{1} & :=\sum_{k=0}^{n} \frac{(n+k) !}{(n-k) !} \frac{(-1)^{k} f^{(n-k)}(1)-(-1)^{n} f^{(n-k)}(-1)}{n ! k ! 2^{n+k+1}}, \quad n=1,2,3, \ldots
\end{aligned}
$$

The coefficients $g_{n}^{0}$ and $g_{n}^{1}$ of the expansion of $g(z)$ and the coefficients $h_{n}^{0}$ and $h_{n}^{1}$ of the expansion of $h(z)$ are defined by similar formulas. The three expansions in (3) converge absolute and uniformly to the respective functions $f(z), g(z)$ and $h(z)$ in $\mathcal{D}_{r}$ (see [6]). The regular case analyzed in [8] corresponds to the particular situation $f_{0}^{0}=f_{0}^{1}, g_{0}^{0}=g_{0}^{1}$ and $h_{0}^{0}=h_{0}^{1}$ (that is equivalent to $f(-1)=$ $g(-1)=h(-1)=0)$.

As it is argued in [8], when $f(-1)=g(-1)=h(-1)=0$, any solution of the differential equation is analytic in $\mathcal{D}_{r}$. But the situation is different when $|f(-1)|+|g(-1)|+|h(-1)| \neq 0$ and we need the following definition.

Definition 1. Denote by $S_{h}$ the linear space of solutions of the homogeneous equation $(z+1) y^{\prime \prime}+$ $f(z) y^{\prime}+g(z) y=0$ that are analytic in $\mathcal{D}_{r}$. Denote by $S$ the affine space of solutions of the complete equation $(z+1) y^{\prime \prime}+f(z) y^{\prime}+g(z) y=h(z)$ that are analytic in $\mathcal{D}_{r}$.

From Frobenius theory we know that the critical exponents of the homogeneous differential equation $(z+1) y^{\prime \prime}+f(z) y^{\prime}+g(z) y=0$ at $z=-1$ are $\mu_{1}=0$ and $\mu_{2}=1-f(-1)$. When $\mu_{2} \notin \mathbb{Z}$ $(f(-1) \notin \mathbb{Z})$, one independent solution of the homogeneous equation is analytic in $\mathcal{D}_{r}$ and the other one is not, as it is of the form $(z+1)^{\mu_{2}} a(z)$ with $a(z)$ analytic in $\mathcal{D}_{r}$. When $\mu_{2}=0,-1,-2, \ldots$, $(f(-1) \in \mathbb{N})$, one independent solution of $(z+1) y^{\prime \prime}+f(z) y^{\prime}+g(z) y=0$ is analytic in $\mathcal{D}_{r}$ and the other one is not, as it is of the form $a_{1}(z) \log (z+1)+(z+1)^{\mu_{2}} a_{2}(z)$ with $a_{1}(z)$ and $a_{2}(z)$ analytic in $\mathcal{D}_{r}$ and $a_{1}(z) \neq 0$ if $\mu_{2}=0$. When $\mu_{2} \in \mathbb{N},(f(-1)=0,-1,-2, \ldots)$, one independent solution of the homogeneous equation is analytic in $\mathcal{D}_{r}$ (and vanishes $\mu_{2}$ times at $z=-1$ ) and the other one is of the 


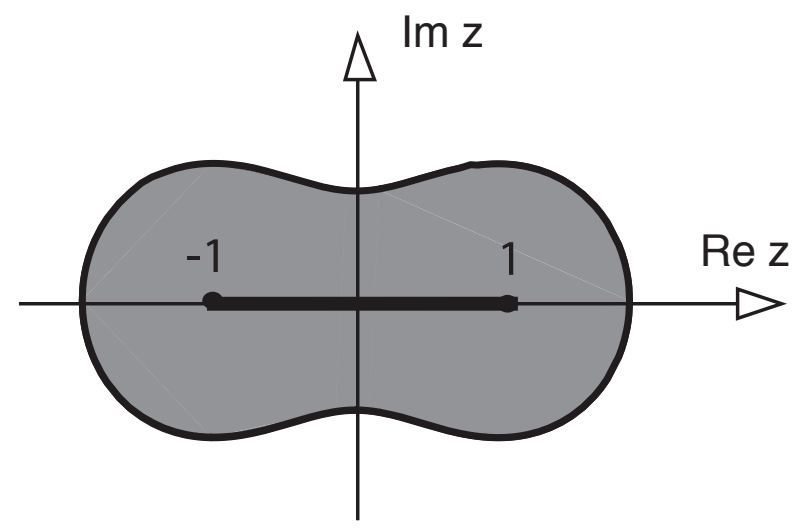

Figure 1: The Cassini disk $\mathcal{D}_{r}=\left\{z \in \mathbb{C}|| z^{2}-1 \mid<r\right\}$ with foci at $z= \pm 1$ and radius $r>1$ contains the real interval $[-1,1]$.

form $(z+1)^{\mu_{2}} a_{1}(z) \log (z+1)+a_{2}(z)$ with $a_{1}(z)$ and $a_{2}(z)$ analytic in $\mathcal{D}_{r}$. Therefore, when $\mu_{2} \in \mathbb{N}$, may be only one or may be two independent solutions of $(z+1) y^{\prime \prime}+f(z) y^{\prime}+g(z) y=0$ analytic at $z=-1$.

From the above discussion we conclude that

$$
\operatorname{dim}\left(S_{h}\right)=\left\{\begin{array}{lll}
1 & \text { when } & f(-1) \neq 0,-1,-2, \ldots \\
1 \text { or } 2 & \text { when } & f(-1)=0,-1,-2, \ldots
\end{array}\right.
$$

On the other hand, it is clear that $S=y_{p}+S_{h}$, where $y_{p}(z)$ is a particular solution of $(z+1) y^{\prime \prime}+$ $f(z) y^{\prime}+g(z) y=h(z)$ that is analytic in $\mathcal{D}_{r}$. The existence of that particular solution $y_{p}(z)$ is not guaranteed "a priori"; then, either $\operatorname{dim}(S)=\operatorname{dim}\left(S_{h}\right)$ or $S$ is empty. (For example, the general solution of the equation $(z+1) y^{\prime \prime}=1$ is $y(z)=c_{1}+c_{2} z+(1+z) \log (z+1) ; c_{1}, c_{2} \in \mathbb{C}$; then $\operatorname{dim}\left(S_{h}\right)=2$ and $S$ is empty. The general solution of the equation $(z+1) y^{\prime \prime}+y^{\prime}=1$ is $y(z)=c_{1}+c_{2} \log (z+1)+z ; c_{1}$, $c_{2} \in \mathbb{C}$; then $\operatorname{dim}\left(S_{h}\right)=\operatorname{dim}(S)=1$.)

Once we have a picture of the spaces $S$ and $S_{h}$ in relation to the value of $f(-1)$, we introduce the key point in the discussion of the paper. Any function $y(z) \in S$ or $y(z) \in S_{h}$ can be written in the form of a two-point Taylor expansion at the base points $z= \pm 1$ (see [6]),

$$
y(z)=\sum_{n=0}^{\infty}\left[a_{n}+b_{n} z\right]\left(z^{2}-1\right)^{n}, \quad z \in \mathcal{D}_{r},
$$

where the coefficients $a_{n}$ and $b_{n}$ are related to the values of the derivatives of $y(z)$ at $z= \pm 1$ in the same form as the coefficients $f_{n}^{0}$ and $f_{n}^{1}$ of $f(z)$ are related to the derivatives of $f(z)$ at $z= \pm 1$ in (4). If we can derive the coefficients $a_{n}$ and $b_{n}$ from $(z+1) y^{\prime \prime}+f(z) y^{\prime}+g(z) y=h(z)$, we will obtain the functions $y \in S$ in the form of a two-point Taylor series (5), when the space $S$ is nonempty. This fact is not guaranteed "a priory"; what is guaranteed is that $S_{h}$ is not empty, moreover, it has dimension at least one. In the regular case $f(-1)=g(-1)=0$, it is guaranteed that the dimension of $S_{h}$ is two (see $[8])$.

As it is not guaranteed "a priory" that $S$ is nonempty, the existence of one analytic solution in $\mathcal{D}_{r}$ of the initial or boundary value problem (1) is not guaranteed "a priory" either; nor even when $h=0$ (homogenous case) or in the regular case $f(-1)=g(-1)=h(-1)=0$. In this paper we analyze the size of the space $S$ and then, the existence and uniqueness of analytic solutions in $\mathcal{D}_{r}$ of the problem (1). We accomplish this task using that any function in $S$ may be written in the form (5): in the remaining of the paper we replace the formal two-point Taylor series in (1) and study if it is possible to obtain the coefficients $a_{n}$ and $b_{n}$ from the differential equation and boundary conditions given in (1). 
For any function $y(z)$ analytic in $\mathcal{D}_{r}$, the series $(5)$ is absolute and uniformly convergent in the interval $[-1,1]$, and we also have $[8]$

$$
\begin{aligned}
y^{\prime}(z) & =\sum_{k=0}^{\infty}\left\{\left[(2 k+1) b_{k}+2(k+1) b_{k+1}\right]+2(k+1) a_{k+1} z\right\}\left(z^{2}-1\right)^{k}, \\
y^{\prime \prime}(z) & =\sum_{k=0}^{\infty} 2(k+1)\left\{\left[(2 k+1) a_{k+1}+2(k+2) a_{k+2}\right]+\left[(2 k+3) b_{k+1}+2(k+2) b_{k+2}\right] z\right\}\left(z^{2}-1\right)^{k},
\end{aligned}
$$

where the convergence of the series is absolute and uniform in the interval $[-1,1]$.

\section{Two-point Taylor expansion representation of the functions of $\mathrm{S}$}

As it happens in the standard Frobenius method for initial value problems, when we replace $f(z), g(z)$ and $h(z)$ by their two-point Taylor expansions (3) in the differential equation $(z+1) y^{\prime \prime}+f(z) y^{\prime}+g(z) y=$ $h(z)$, and the solution $y(z)$ and its derivatives by their two-point Taylor expansions (5) and (6), we find that the coefficients $a_{n}$ and $b_{n}$ satisfy, for $n=0,1,2, \ldots$, a linear system of two recurrences:

$$
\begin{aligned}
& \begin{array}{l}
4(n+1)(n+2)\left(a_{n+2}+b_{n+2}\right)+2(n+1)(2 n+1) a_{n+1}+2(n+1)(4 n+3) b_{n+1}+2 n(2 n+1) b_{n} \\
+\sum_{k=0}^{n}\left\{f_{n-k}^{0}\left[(2 k+1) b_{k}+2(k+1) b_{k+1}\right]+2(k+1)\left(f_{n-k}^{1}+f_{n-k-1}^{1}\right) a_{k+1}\right. \\
\left.\quad+\left(g_{n-k}^{1}+g_{n-k-1}^{1}\right) b_{k}+g_{n-k}^{0} a_{k}\right\}=h_{n}^{0},
\end{array} \\
& \begin{array}{l}
4(n+1)(n+2)\left(a_{n+2}+b_{n+2}\right)+2(n+1)(2 n+1) a_{n+1}+2(n+1)(2 n+3) b_{n+1} \\
+\sum_{k=0}^{n}\left\{f_{n-k}^{1}\left[(2 k+1) b_{k}+2(k+1) b_{k+1}\right]+2(k+1) f_{n-k}^{0} a_{k+1}+g_{n-k}^{0} b_{k}+g_{n-k}^{1} a_{k}\right\}=h_{n}^{1},
\end{array}
\end{aligned}
$$

with $f_{-1}^{0}=g_{-1}^{0}=f_{-1}^{1}=g_{-1}^{1}=0$. It is not possible to solve this linear system of equations for $a_{n+2}$ and $b_{n+2}$. We solve this technical difficulty by retaining the second equation and replacing the first one by the difference between the two equations with $n$ replaced by $n+1$. In this way we obtain, for $n=0,1,2, \ldots$, the linear non homogeneous system (homogeneous when $h=0$ ):

$$
\begin{aligned}
& 4(n+1)(n+2)\left(a_{n+2}+b_{n+2}\right)+2(n+1)(2 n+1) a_{n+1}+2(n+1)(2 n+3) b_{n+1} \\
& +\sum_{k=0}^{n}\left\{f_{n-k}^{1}\left[(2 k+1) b_{k}+2(k+1) b_{k+1}\right]+2(k+1) f_{n-k}^{0} a_{k+1}+g_{n-k}^{0} b_{k}+g_{n-k}^{1} a_{k}\right\}=h_{n}^{1}, \\
& 2(n+2)\left[2(n+1)+\left(f_{0}^{0}-f_{0}^{1}\right)\right] b_{n+2}-2(n+2)\left(f_{0}^{0}-f_{0}^{1}\right) a_{n+2}+2(n+1)(n+2) b_{n+1} \\
& +\sum_{k=0}^{n+1}\left[(2 k+1)\left(f_{n-k+1}^{0}-f_{n-k+1}^{1}\right) b_{k}+2(k+1) f_{n-k}^{1} a_{k+1}+\left(g_{n-k+1}^{0}-g_{n-k+1}^{1}\right)\left(a_{k}-b_{k}\right)+g_{n-k}^{1} b_{k}\right] \\
& +\sum_{k=0}^{n}\left[2(k+1)\left(f_{n-k+1}^{0}-f_{n-k+1}^{1}\right) b_{k+1}+2(k+1)\left(f_{n-k+1}^{1}-f_{n-k+1}^{0}\right) a_{k+1}\right]=h_{n+1}^{0}-h_{n+1}^{1} .
\end{aligned}
$$

Then, in general, as it happens in the standard Frobenius method or in the particular regular boundary problem analyzed in [8], the computation of the coefficients $a_{n}$ and $b_{n}$ involves the previous coefficients $a_{0}, b_{0}, \ldots, a_{n-1}$ and $b_{n-1}$. But we find here a particularity that we do not find in the regular problem solved in [8]: in general, for a given $n=0,1,2, \ldots$, we can solve the linear system (8) for $a_{n+2}$ and $b_{n+2}$ if and only if

$$
\left|\begin{array}{cc}
1 & 1 \\
f_{0}^{1}-f_{0}^{0} & 2(n+1)+f_{0}^{0}-f_{0}^{1}
\end{array}\right| \neq 0 \Leftrightarrow f(-1) \equiv f_{0}^{0}-f_{0}^{1} \neq-n-1 .
$$


Then, if $-f(-1) \notin \mathbb{N}$, we can solve the linear system (8) for $a_{n+2}$ and $b_{n+2}$ for any $n=0,1,2, \ldots$ But if $-f(-1) \equiv n_{0} \in \mathbb{N}$, then we can solve the system (8) for $a_{n+2}$ and $b_{n+2}$ for any $n=0,1,2, \ldots$, except for $n=n_{0}-1$. For convenience, when $-f(-1) \notin \mathbb{N}$ we define $n_{0}=0$.

Therefore, in any case, we can solve the linear system (8) for $a_{n+2}$ and $b_{n+2}$ for $n=n_{0}, n_{0}+$ $1, n_{0}+2, \ldots$ This means that we obtain all the coefficients $a_{n}$ and $b_{n}$ required to represent $y(z)$ in the form (5) for $n=n_{0}+2, n_{0}+3, n_{0}+4, \ldots$, as a function of the first $2\left(n_{0}+2\right)$ coefficients $a_{0}, b_{0}, a_{1}, b_{1}, \ldots, a_{n_{0}+1}, b_{n_{0}+1}$. But these $2\left(n_{0}+2\right)$ first coefficients are not totally free, as they must satisfy the equations (8) for $n=0,1,2, \ldots, n_{0}-1$. Moreover, they must also satisfy the second equation in (8) for $n=-1$ (empty sums being understood as zero), since in passing from (7) to (8) we missed this equation when we replaced $n$ by $n+1$ (this is equivalent to the compatibility condition of the equations $(7)$ at $n=0$ ). These facts impose $2 n_{0}+1$ linear restrictions (not all of them necessarily independent) to the $2\left(n_{0}+2\right)$ first coefficients $a_{0}, b_{0}, a_{1}, b_{1}, \ldots, a_{n_{0}+1}, b_{n_{0}+1}$. Let's denote these equations by $L_{k}\left[a_{0}, b_{0}, a_{1}, b_{1}, \ldots, a_{n_{0}+1}, b_{n_{0}+1}\right]=0, k=1,2,3, \ldots, 2 n_{0}+1$, where, if $k$ is odd, $L_{k}$ is the second equation of system (8) for $n=(k-3) / 2$, and if $k$ is even, $L_{k}$ is the first equation of system (8) for $n=(k-2) / 2$. In general, this set of equations is non homogeneous; it is homogenous when $h(z)=0$.

In the particular case of the regular problem analyzed in [8] we have that $n_{0}=0$, as $f(-1)=0$. Then, we can obtain from system (8) all the coefficients $a_{n}$ and $b_{n}$ for $n \geq 2$ as a function of the first four coefficients $a_{0}, b_{0}, a_{1}$ and $b_{1}$. In this case, the above mentioned set of restrictions reduces to only one restriction $L_{1}\left[a_{0}, b_{0}, a_{1}, b_{1}\right]=0$ (the second equation in (8) for $n=-1$ ). But using that $f(-1)=g(-1)=h(-1)=0$ we see that this equation is the tautology $0=0$ and then, it does not introduce any linear dependence between the coefficients $a_{0}, b_{0}, a_{1}$ and $b_{1}$.

As a difference with the Frobenius method where we only have one recurrence for the sequence of standard Taylor coefficients, here we have a system of two recurrences (8). But moreover, the computation of the coefficients $a_{n}, b_{n}$ for $n \geq n_{0}+2$ requires the initial seed $a_{0}, b_{0}, a_{1}, b_{1}, \ldots, a_{n_{0}+1}, b_{n_{0}+1}$. These $2 n_{0}+4$ coefficients satisfy the above mentioned $2 n_{0}+1$ equations. This does not mean that the linear space $S_{h}$ or the affine space $S$ may have dimension three or more, these spaces have, of course, dimension at most two. It is happening that, apart from the affine space $S$ of (true) solutions of $(z+1) y^{\prime \prime}+f(z) y^{\prime}+g(z) y=h(z)$, there is a bigger space of formal solutions $W$ defined by:

$$
\begin{gathered}
W:=\left\{y(z)=\sum_{n=0}^{\infty}\left[a_{n}+b_{n} z\right]\left(z^{2}-1\right)^{n} \mid a_{n}, b_{n} \text { given in }(8) \text { for } n \geq n_{0}+2\right. \\
\left(a_{0}, b_{0}, a_{1}, b_{1}, \ldots, a_{n_{0}+1}, b_{n_{0}+1}\right) \in \mathbb{C}^{2 n_{0}+4} \\
\left.\quad \text { with } L_{k}\left[a_{0}, b_{0}, \ldots, a_{n_{0}+1}, b_{n_{0}+1}\right]=0, k=1,2,3, \ldots, 2 n_{0}+1\right\} .
\end{gathered}
$$

Formally, all the two-point Taylor series in $W$ are solutions of $(z+1) y^{\prime \prime}+f(z) y^{\prime}+g(z) y=h(z)$. But not all of them are convergent, only a subset: the affine space $S$ of (true) solutions of $(z+1) y^{\prime \prime}+$ $f(z) y^{\prime}+g(z) y=h(z)$, that may be written in the form:

$$
S=\left\{y \in W \mid \sum_{n=0}^{\infty}\left[a_{n}+b_{n} z\right]\left(z^{2}-1\right)^{n} \text { is uniformly convergent in }[-1,1]\right\} .
$$

In the following section we derive a more practical characterization of the space $S$ in the form of two extra linear equations for the coefficients $a_{0}, b_{0}, a_{1}, b_{1}, \ldots, a_{n_{0}+1}, b_{n_{0}+1}$. This characterization lets us give some more precise information about the size of the space $S$. 


\section{Algebraic characterization of the space $\mathrm{S}$}

From (8) and the discussion below that formula, we see that we may solve (8) for $\left(a_{n}, b_{n}\right)$ for $n \geq n_{0}+2$ in the schematic form

$$
\begin{aligned}
a_{n} & =\sum_{k=0}^{n-1}\left[A_{n, k} a_{k}+B_{n, k} b_{k}\right]+E_{n}, \\
b_{n} & =\sum_{k=0}^{n-1}\left[C_{n, k} a_{k}+D_{n, k} b_{k}\right]+F_{n},
\end{aligned}
$$

where the coefficients $A_{n, k}, B_{n, k}, C_{n, k}$ and $D_{n, k}$ are functions of $f_{k}^{0}, f_{k}^{1}, g_{k}^{0}$ and $g_{k}^{1}$. The coefficients $E_{n, k}$ and $F_{n, k}$ are functions of $h_{k}^{0}$ and $h_{k}^{1}, k=0,1,2, \ldots, n-1$. For simplicity, we do not detail here these functions, as the precise value of these coefficients is not needed in the theoretical discussion. It is not needed either in computation in the particular examples, as it is more convenient the use of an algebraic manipulator to compute $\left(a_{n}, b_{n}\right), n \geq n_{0}+2$, directly from (8).

For a fixed $m \in \mathbb{N}, m \geq 2 n_{0}+2$, and $n=0,1,2, \ldots, m-n_{0}-1$, we define the vectors:

$$
v_{n}:=\left(a_{n+n_{0}+2-m}, b_{n+n_{0}+2-m}, a_{n+n_{0}+3-m}, b_{n+n_{0}+3-m}, \ldots, a_{n+n_{0}}, b_{n+n_{0}}, a_{n+n_{0}+1}, b_{n+n_{0}+1}\right) \in \mathbb{C}^{2 m},
$$

with $a_{-k}=b_{-k}=0$ for $k \in \mathbb{N}$. In particular we have:

$$
v_{m-n_{0}-2}=\left(a_{0}, b_{0}, a_{1}, b_{1}, \ldots, a_{m-1}, b_{m-1}\right) \quad \text { and } \quad v_{0}=\left(0,0, \ldots, 0,0, a_{0}, b_{0}, a_{1}, b_{1}, \ldots, a_{n_{0}+1}, b_{n_{0}+1}\right) .
$$

For $n=0,1,2, \ldots, m-n_{0}-2$, define the $(2 m) \times(2 m)$ matrix

$$
M_{n}:=\left(\begin{array}{ccccccccc}
0 & 0 & 1 & 0 & 0 & 0 & 0 & \ldots & 0 \\
0 & 0 & 0 & 1 & 0 & 0 & 0 & \ldots & 0 \\
\ldots & \ldots & \ldots & \ldots & \ldots & \ldots & \ldots & \ldots & \ldots \\
\ldots & \ldots & \ldots & \ldots & \ldots & \ldots & \ldots & \ldots & \ldots \\
0 & 0 & 0 & 0 & 0 & \ldots & 0 & 1 & 0 \\
0 & 0 & 0 & 0 & 0 & \ldots & 0 & 0 & 1 \\
0 & \ldots & 0 & A_{n+n_{0}+2,0} & B_{n+n_{0}+2,0} & \ldots & \ldots & A_{n+n_{0}+2, n+n_{0}+1} & B_{n+n_{0}+2, n+n_{0}+1} \\
0 & \ldots & 0 & C_{n+n_{0}+2,0} & D_{n+n_{0}+2,0} & \ldots & \ldots & C_{n+n_{0}+2, n+n_{0}+1} & D_{n+n_{0}+2, n+n_{0}+1}
\end{array}\right) .
$$

The only non-null elements of this matrix are the corresponding to the entries $m_{i, i+2}=1, i=$ $1,2,3, \ldots, 2 m-2$, and to the entries $m_{2 m-1, k}, m_{2 m, k}, k=0,1,2, \ldots, n+n_{0}+1$. In particular we have

$$
M_{0}=\left(\begin{array}{ccccccccc}
0 & 0 & 1 & 0 & 0 & 0 & 0 & \ldots & 0 \\
0 & 0 & 0 & 1 & 0 & 0 & 0 & \ldots & 0 \\
\ldots & \ldots & \ldots & \ldots & \ldots & \ldots & \ldots & \ldots & \ldots \\
\ldots & \ldots & \ldots & \ldots & \ldots & \ldots & \ldots & \ldots & \ldots \\
0 & 0 & 0 & 0 & 0 & \ldots & 0 & 1 & 0 \\
0 & 0 & 0 & 0 & 0 & \ldots & 0 & 0 & 1 \\
0 & \ldots & 0 & A_{n_{0}+2,0} & B_{n_{0}+2,0} & \ldots & \ldots & A_{n_{0}+2, n_{0}+1} & B_{n_{0}+2, n_{0}+1} \\
0 & \ldots & 0 & C_{n_{0}+2,0} & D_{n_{0}+2,0} & \ldots & \ldots & C_{n_{0}+2, n_{0}+1} & D_{n_{0}+2, n_{0}+1}
\end{array}\right)
$$

and

$$
M_{m-n_{0}-2}=\left(\begin{array}{ccccccccc}
0 & 0 & 1 & 0 & 0 & 0 & 0 & \ldots & 0 \\
0 & 0 & 0 & 1 & 0 & 0 & 0 & \ldots & 0 \\
\ldots & \ldots & \ldots & \ldots & \ldots & \ldots & \ldots & \ldots & \ldots \\
\ldots & \ldots & \ldots & \ldots & \ldots & \ldots & \ldots & \ldots & \ldots \\
0 & 0 & 0 & 0 & 0 & \ldots & 0 & 1 & 0 \\
0 & 0 & 0 & 0 & 0 & \ldots & 0 & 0 & 1 \\
A_{m, 0} & B_{m, 0} & A_{m, 1} & B_{m, 1} & \ldots & \ldots & \ldots & A_{m, m-1} & B_{m, m-1} \\
C_{m, 0} & D_{m, 0} & C_{m, 1} & D_{m, 1} & \ldots & \ldots & \ldots & C_{m, m-1} & D_{m, m-1}
\end{array}\right)
$$


We also need, for $n=0,1,2, \ldots, m-n_{0}-2$, the definition of the vector

$$
c_{n}:=\left(0,0, \ldots, 0,0, E_{n+2}, F_{n+2}\right) \in \mathbb{C}^{2 m} .
$$

Then, the system of recurrences (9) (that indeed represents (8)) can be written in matrix form

$$
v_{n}=M_{n-1} v_{n-1}+c_{n-1}, \quad n=1,2,3, \ldots, m-n_{0}-1 .
$$

To find the solution of this linear recurrence for the vector $v_{n}$, we define recurrently the following matrices

$$
\begin{aligned}
\mathcal{M}_{0} & :=M_{0}, \quad \mathcal{M}_{n}:=M_{n} \mathcal{M}_{n-1}, \\
\mathcal{C}_{0} & :=c_{0}, \quad \mathcal{C}_{n}:=M_{n} \mathcal{C}_{n-1}+c_{n}, \quad n=1,2,3, \ldots, m-n_{0}-2,
\end{aligned}
$$

or equivalently,

$$
\mathcal{M}_{n}=\prod_{k=0}^{n} M_{n-k}, \quad \mathcal{C}_{n}=c_{n}+\sum_{k=0}^{n-1}\left[M_{n} \cdot M_{n-1} \cdots M_{k+1}\right] c_{k}, \quad n=0,1,2,3, \ldots, m-n_{0}-2 .
$$

Then, we find

$$
v_{m-n_{0}-1}=\mathcal{M}_{m-n_{0}-2} v_{0}+\mathcal{C}_{m-n_{0}-2},
$$

or, in an extended form:

$$
\begin{aligned}
& \left(\begin{array}{c}
\star \\
\star \\
\cdot \\
\cdot \\
\cdot \\
\star \\
\star \\
a_{m} \\
b_{m}
\end{array}\right)=\left(\begin{array}{c}
\star \\
\star \\
\cdot \\
\cdot \\
\star \\
\star \\
\mathcal{B}_{2 m-1} \\
\mathcal{B}_{2 m}
\end{array}\right) \\
& +\left(\begin{array}{cccccccc}
\star & \star & \star & \star & \star & \ldots & \star & \star \\
\star & \star & \star & \star & \star & \ldots & \star & \star \\
\ldots & \ldots & \ldots & \ldots & \ldots & \ldots & \ldots & \ldots \\
\ldots & \ldots & \ldots & \ldots & \ldots & \ldots & \ldots & \ldots \\
\cdots & \ldots & \ldots & \ldots & \star & \ldots & \ldots & \ldots \\
\star & \star & \star & \star & \star & \ldots & \star & \star \\
\star & \star & \star & \star & \ldots & \star \\
\star & \ldots & \star & \mathcal{M}_{2 m-1,2 m-2 n_{0}-3} & \mathcal{M}_{2 m-1,2 m-2 n_{0}-2} & \ldots & \mathcal{M}_{2 m-1,2 m-1} & \mathcal{M}_{2 m-1,2 m} \\
\star & \ldots & \star & \mathcal{M}_{2 m, 2 m-2 n_{0}-3} & \mathcal{M}_{2 m, 2 m-2 n_{0}-2} & \ldots & \mathcal{M}_{2 m, 2 m-1} & \mathcal{M}_{2 m, 2 m}
\end{array}\right)\left(\begin{array}{c}
0 \\
. \\
0 \\
a_{0} \\
b_{0} \\
\cdot \\
\cdot \\
a_{n_{0}+1} \\
b_{n_{0}+1}
\end{array}\right),
\end{aligned}
$$

where $\mathcal{M}_{i, j}$ are the entrances of the last two rows and last $2 n_{0}+4$ columns of the matrix $\mathcal{M}_{m-n_{0}-2}, \mathcal{B}_{i}$ are the last two components of the vector $\mathcal{C}_{m-n_{0}-2}$ and the $\star$ denote complex (unspecified) numbers. The two-point Taylor series of an analytic function in $\mathcal{D}_{r}$ converges in $[-1,1]$ if it converges at $z=0[6]$. And it converges at $z=0$ if and only if $\lim _{m \rightarrow \infty}\left(a_{m}, b_{m}\right)=(0,0)$. Then, taking the limit $m \rightarrow \infty$ into the above equation we find

$$
\left(\begin{array}{c}
\star \\
\star \\
\cdot \\
\cdot \\
\cdot \\
\star \\
\star \\
0 \\
0
\end{array}\right)=\left(\begin{array}{cccccccc}
\star & \star & \star & \star & \star & \ldots & \star & \star \\
\star & \star & \star & \star & \star & \ldots & \star & \star \\
\ldots & \ldots & \ldots & \ldots & \ldots & \ldots & \ldots & \ldots \\
\ldots & \ldots & \ldots & \ldots & \ldots & \ldots & \ldots & \ldots \\
\ldots & \ldots & \ldots & \ldots & \ldots & \ldots & \ldots & \ldots \\
\star & \star & \star & \star & \star & \ldots & \star & \star \\
\star & \star & \star & \star & \star & \ldots & \star & \star \\
\star & \ldots & \star & H_{1,1} & H_{1,2} & \ldots & H_{1,2 n_{0}+3} & H_{1,2 n_{0}+4} \\
\star & \ldots & \star & H_{2,1} & H_{2,2} & \ldots & H_{2,2 n_{0}+3} & H_{2,2 n_{0}+4}
\end{array}\right)\left(\begin{array}{c}
0 \\
\cdot \\
0 \\
a_{0} \\
b_{0} \\
\cdot \\
\cdot \\
a_{n_{0}+1} \\
b_{n_{0}+1}
\end{array}\right)+\left(\begin{array}{c}
\star \\
\star \\
\cdot \\
\cdot \\
\cdot \\
\star \\
\gamma_{1} \\
\gamma_{2}
\end{array}\right),
$$


where we have denoted

$$
\begin{aligned}
H_{i, j} & :=\lim _{m \rightarrow \infty} \mathcal{M}_{2 m+i-2,2 m-2 n_{0}+j-4}, \quad i=1,2, \quad j=1,2,3, \ldots, 2 n_{0}+4, \\
\gamma_{1} & =\lim _{m \rightarrow \infty} \mathcal{B}_{2 m-1}, \quad \gamma_{2}=\lim _{m \rightarrow \infty} \mathcal{B}_{2 m} .
\end{aligned}
$$

Then, the two equations that we were looking for are, for $k=1,2$ :

$$
H_{k}\left[a_{0}, b_{0}, \ldots, a_{n_{0}+1}, b_{n_{0}+1}\right]:=H_{k, 1} a_{0}+H_{k, 2} b_{0}+\cdots+H_{k, 2 n_{0}+3} a_{n_{0}+1}+H_{k, 2 n_{0}+4} b_{n_{0}+1}+\gamma_{k}=0 .
$$

Therefore, at this moment, we have found the more practical characterization of the space $S$ of true solutions of $(z+1) y^{\prime \prime}+f(z) y^{\prime}+g(z) y=h(z)$ that we were looking for:

$$
\begin{aligned}
S:=\{ & y(z)=\sum_{n=0}^{\infty}\left[a_{n}+b_{n} z\right]\left(z^{2}-1\right)^{n} \mid a_{n}, b_{n} \text { given in }(8) \text { for } n \geq n_{0}+2 ; \\
& \left(a_{0}, b_{0}, a_{1}, b_{1}, \ldots, a_{n_{0}+1}, b_{n_{0}+1}\right) \in \mathbb{C}^{2 n_{0}+4} \text { with } L_{k}\left[a_{0}, b_{0}, a_{1}, b_{1}, \ldots, a_{n_{0}+1}, b_{n_{0}+1}\right]=0 \\
& \text { for } \left.k=1,2,3, \ldots, 2 n_{0}+1, \text { and } H_{k}\left[a_{0}, b_{0}, a_{1}, b_{1}, \ldots, a_{n_{0}+1}, b_{n_{0}+1}\right]=0 \text { for } k=1,2\right\} .
\end{aligned}
$$

In other words, the coefficients $a_{0}, b_{0}, a_{1}, b_{1}, \ldots, a_{n_{0}+1}, b_{n_{0}+1}$ of the two-point Taylor expansion of any function in $S$ must be a solution of the following linear system of $2 n_{0}+3$ equations:

$$
\begin{cases}L_{k}\left[a_{0}, b_{0}, a_{1}, b_{1}, \ldots, a_{n_{0}+1}, b_{n_{0}+1}\right]=0, & k=1,2,3, \ldots, 2 n_{0}+1, \\ H_{k}\left[a_{0}, b_{0}, a_{1}, b_{1}, \ldots, a_{n_{0}+1}, b_{n_{0}+1}\right]=0, & k=1,2 .\end{cases}
$$

This system is homogenous when $h=0$ (when $h_{n}^{0}=h_{n}^{1}=0$ ) and non-homogenous when $h \neq 0$. Let's denote $(15)_{h}$ the system (15) when $h=0$. We know that $\operatorname{dim}\left(S_{h}\right)=1$ or 2 . This means that $\operatorname{rank}\left[(15)_{h}\right]=2 n_{0}+2$ or $2 n_{0}+3$ and then, the homogeneous system has a one or two-dimensional space of solutions. On the other hand, we know that $\operatorname{dim}(S)=1$ or 2 , or $S$ is empty. This means that there are three possibilities:

(i) $\operatorname{rank}[(15)]=\operatorname{rank}\left[(15)_{h}\right]=2 n_{0}+2 ;$ then $\operatorname{dim}(S)=\operatorname{dim}\left(S_{h}\right)=2$.

(ii) $\operatorname{rank}[(15)]=\operatorname{rank}\left[(15)_{h}\right]=2 n_{0}+3$; then $\operatorname{dim}(S)=\operatorname{dim}\left(S_{h}\right)=1$.

(iii) $\operatorname{rank}[(15)]=2 n_{0}+3$ and $\operatorname{rank}\left[(15)_{h}\right]=2 n_{0}+2$; then $\operatorname{dim}\left(S_{h}\right)=2$ and $S$ is empty.

Therefore, when $\operatorname{rank}\left[(15)_{h}\right]=2 n_{0}+3, S$ is nonempty and $\operatorname{dim}(S)=\operatorname{dim}\left(S_{h}\right)=1$. When $\operatorname{rank}\left[(15)_{h}\right]=2 n_{0}+2$, then either, $\operatorname{dim}(S)=2$ or $S$ is empty.

When $f(-1) \neq 0,-1,-2, \ldots$, we know "a priory", without need of studying the ranks of the system $(15)$, that $\operatorname{dim}\left(S_{h}\right)=\operatorname{dim}(S)=1$. On the other hand, when $f(-1)=0,-1,-2, \ldots$, we do not know "a priory" the dimensions, we need to compute the above mentioned ranks. The exception is, of course, the homogeneous case $h=0$, because the complete and homogeneous systems are the same one. Then $S=S_{h}$, and we know "a priory" that there is, at least, a one dimensional space of analytic solutions in $\mathcal{D}_{r}$ of the homogeneous equation. Another exception is the regular case, as we know "a priory" that $\operatorname{dim}(S)=2$ (it is proved in [8] that the only two equations $H_{k}=0$ that define $S$ in this case are linearly independent).

\subsection{Polynomial coefficients}

When the coefficient functions $f, g$ and $h$ are polynomials, we can simplify the formulation of the above existence and uniqueness criterion. In general, the computation of the coefficients $\left(a_{n}, b_{n}\right)$ requires a matrix $M_{n}$ of size $(2 m) \times(2 m)$ with $m \geq n+n_{0}+2$. This means that we need matrices of increasing size to compute the coefficients when $n$ increases. In the case of polynomial coefficients, the situation is different. The recurrences (8) are of constant order $s$ independent of $n$ and the computation of the 
coefficients $a_{n}$ and $b_{n}$ involves only the previous $2 s$ coefficients $a_{n-s}, b_{n-s}, \ldots, a_{n-1}$ and $b_{n-1}$. Thus, in this case, we do not need matrices of increasing size, but matrices of constant size $(2 s) \times(2 s)$.

The recurrence system (8) for polynomial coefficients is of the form:

$$
\begin{aligned}
& a_{n}=\sum_{k=n-s}^{n-1}\left[A_{n, k} a_{k}+B_{n, k} b_{k}\right]+E_{n}, \\
& b_{n}=\sum_{k=n-s}^{n-1}\left[C_{n, k} a_{k}+D_{n, k} b_{k}\right]+F_{n},
\end{aligned}
$$

for a certain $s \in \mathbb{N}, n=n_{0}, n_{0}+1, n_{0}+2, \ldots$, with $a_{-k}=b_{-k}=0, k \in \mathbb{N}$. The discussion is identical to the one developed in the general case analyzed above, but now we can eliminate the restriction $n \leq m-n_{0}-2$. Moreover, we can simplify the computations because now, the size of the matrices $M_{n}$ does not depend on $n$. We can now define the matrices $M_{n}$ of fixed size $(2 s) \times(2 s)$ in the form:

$$
M_{n}:=\left(\begin{array}{ccccccccc}
0 & 0 & 1 & 0 & 0 & 0 & 0 & \ldots & 0 \\
0 & 0 & 0 & 1 & 0 & 0 & 0 & \ldots & 0 \\
\ldots & \ldots & \ldots & \ldots & \ldots & \ldots & \ldots & \ldots & \ldots \\
\ldots & \ldots & \ldots & \ldots & \ldots & \ldots & \ldots & \ldots & \ldots \\
0 & 0 & 0 & 0 & 0 & \ldots & 0 & 1 & 0 \\
A_{n+2, n+2-s} & B_{n+2, n+2-s} & \ldots & A_{n+2,0} & B_{n+2,0} & \ldots & \ldots & A_{n+2, n+1} & B_{n+2, n+1} \\
C_{n+2, n+2-s} & D_{n+2, n+2-s} & \ldots & C_{n+2,0} & D_{n+2,0} & \ldots & \ldots & C_{n+2, n+1} & D_{n+2, n+1}
\end{array}\right)
$$

instead of the form (10), with $A_{n,-k}=B_{n,-k}=C_{n,-k}=D_{n,-k}=0$ for $k \in \mathbb{N}$. The computation of the system (15) is identical. The only difference is that now, the matrices $\mathcal{M}_{m}$ are of size $(2 s) \times(2 s)$ $\forall m \in \mathbb{N}$ and the vectors $\mathcal{C}_{m} \in \mathbb{R}^{2 s} \forall m \in \mathbb{N}$.

\section{Existence and uniqueness criterion for the boundary value pro- blem (1)}

Once we have the algebraic description (14) of the space $S$ of solutions analytic in $\mathcal{D}_{r}$ of the equation $(z+1) y^{\prime \prime}+f(z) y^{\prime}+g(z) y=h(z)$, we focus our attention on the boundary value problem (1) stated in the introduction. Then, we introduce in the study the two boundary conditions given in (1), in order to find an algebraic description of the solutions of (1). From (5) and (6) we have

$$
\left(\begin{array}{c}
y(-1) \\
y(1) \\
y^{\prime}(-1) \\
y^{\prime}(1)
\end{array}\right)=T\left(\begin{array}{l}
a_{0} \\
b_{0} \\
a_{1} \\
b_{1}
\end{array}\right)
$$

where $T$ is the regular matrix

$$
T=\left(\begin{array}{cccc}
1 & -1 & 0 & 0 \\
1 & 1 & 0 & 0 \\
0 & 1 & -2 & 2 \\
0 & 1 & 2 & 2
\end{array}\right)
$$

(The first four coefficients $a_{0}, b_{0}, a_{1}, b_{1}$ of the two-point Taylor expansion (5) are related to $y(-1)$, $y(1), y^{\prime}(-1), y^{\prime}(1)$ by the matrix $\left.T^{-1}\right)$. Write the matrix $B T$, where $B$ is the $2 \times 4$ matrix defining the boundary condition in (1), in the form

$$
B T=\left(\begin{array}{llll}
R_{1,1} & R_{1,2} & R_{1,3} & R_{1,4} \\
R_{2,1} & R_{2,2} & R_{2,3} & R_{2,4}
\end{array}\right)
$$


Then, the boundary value problem (1) may be written in the following equivalent form that stresses the role of the first four coefficients of the two-point Taylor expansion of $y(x)$ in the boundary value equations:

$$
\left\{\begin{array}{l}
(x+1) y^{\prime \prime}+f(x) y^{\prime}+g(x) y=h(z) \text { in }(-1,1), \\
R_{1}\left[a_{0}, b_{0}, a_{1}, b_{1}\right]:=R_{1,1} a_{0}+R_{1,2} b_{0}+R_{1,3} a_{1}+R_{1,4} b_{1}-\alpha=0, \\
R_{2}\left[a_{0}, b_{0}, a_{1}, b_{1}\right]:=R_{2,1} a_{0}+R_{2,2} b_{0}+R_{2,3} a_{1}+R_{2,4} b_{1}-\beta=0 .
\end{array}\right.
$$

When we add the above two algebraic equations $R_{1}$ and $R_{2}$ to the set of equations (15) that describe the space $S$ of solutions of $(x+1) y^{\prime \prime}+f(x) y^{\prime}+g(x) y=h(x)$, we find that the coefficients $a_{0}, b_{0}, \ldots, a_{n_{0}+1}, b_{n_{0}+1}$ of the two-point Taylor solutions $y(x)$ of (20) (if any) are solutions of the algebraic linear system:

$$
\begin{cases}L_{k}\left[a_{0}, b_{0}, a_{1}, b_{1}, \ldots, a_{n_{0}+1}, b_{n_{0}+1}\right]=0, & k=1,2,3, \ldots, 2 n_{0}+1, \\ H_{k}\left[a_{0}, b_{0}, a_{1}, b_{1}, \ldots, a_{n_{0}+1}, b_{n_{0}+1}\right]=0, & k=1,2, \\ R_{k}\left[a_{0}, b_{0}, a_{1}, b_{1}\right]=0, & k=1,2 .\end{cases}
$$

The remaining coefficients $a_{n}, b_{n}$ for $n \geq n_{0}+2$ are obtained recursively from (8). The system (21) is a linear system of $2 n_{0}+5$ equations with $2 n_{0}+4$ unknowns (in the regular case, the system reduces to the last 4 equations). The existence and uniqueness of solutions of the system (21) is equivalent to the existence and uniqueness of solution of the problem (20). Then, we can finally formulate the following existence and uniqueness criterion for the boundary value problem (1):

\section{Existence and uniqueness criterion}

(i) If the system (21) has not a solution, then problem (1) has not an analytic solution in $\mathcal{D}_{r}$.

(ii) If the system (21) has a unique solution, then problem (1) has a unique analytic solution in $\mathcal{D}_{r}$.

(iii) If the system (21) has a one-dimensional space of solutions, then problem (1) has a onedimensional family of analytic solutions in $\mathcal{D}_{r}$.

(iv) If the system (21) has a two-dimensional space of solutions, then problem (1) has a twodimensional family of analytic solutions in $\mathcal{D}_{r}$.

Observation 1. According to the ranks of $(15)$ and $(15)_{h}$ we have that:

1. If $\operatorname{rank}[(15)]=\operatorname{rank}\left[(15)_{h}\right]=2 n_{0}+3$ (this is granted when $f(-1) \neq 0,-1,-2, \ldots$ ), then (iv) is not possible.

2. If $\operatorname{rank}[(15)]=2 n_{0}+3$ and $\operatorname{rank}\left[(15)_{h}\right]=2 n_{0}+2$ (necessarily $\left.f(-1)=0,-1,-2, \ldots\right)$; then only (i) is possible.

Observation 2. In practice, the coefficients of the two equations $H_{k}$ in system (21) are computed approximately, as the limits of the matrices $\mathcal{M}_{m}$ involved in their computation can be computed only approximately (see (12) and (13)). Therefore, the above existence and uniqueness criterion for solution of (1) is useful when the systems (15) and $(15)_{h}$ are well conditioned. In order to determine the rank of these systems in practice, it is convenient to compute the matrices $\mathcal{M}_{m}$ up to an index $m$ at which the rank of the systems $(15)$ and $(15)_{h}$ stabilize. On the other hand, the criterion is constructive as it provides an approximation to the solution of the form (5) once the coefficients $\left(a_{0}, b_{0}, \ldots, a_{n_{0}+1}, b_{n_{0}+1}\right)$ are computed from (21).

Observation 3. When $f(-1) \neq 0,-1,-2, \ldots$, the rank of the first $2 n_{0}+3$ equations in (21) is $2 n_{0}+3$. When $f(-1)=g(-1)=h(-1)=0$ (regular case), the system (21) consists only of the four last equations and the rank of the two equations $H_{k}=0, k=1,2$, is two. In any other case, the rank of the first $2 n_{0}+3$ equations in the system (21) is not known a priori; it is calculated once we have computed the first $2 n_{0}+3$ equations of system (21). 
The key point in the discussion of the dimensions of $S$ and $S_{h}$ is system (15), and the key point in the discussion of the existence and uniqueness of problem (1) is system (21). In the examples of the following section we show how these systems are computed in practice and how the above criterium of existence and uniqueness may be implemented.

\section{Examples}

In the examples of this section we explore all the possible situations in relation to the value of $f(-1)$ and the sizes of the spaces $S$ and $S_{h}$ :

(i) $f(-1) \neq 0,-1,-2, \ldots$, that is, $\operatorname{dim}(S)=\operatorname{dim}\left(S_{h}\right)=1$. Example 1 .

(ii) $f(-1)=0,-1,-2, \ldots$ and $\operatorname{dim}(S)=\operatorname{dim}\left(S_{h}\right)=1$. Example 2 .

(iii) $f(-1)=0,-1,-2, \ldots$ and $\operatorname{dim}(S)=\operatorname{dim}\left(S_{h}\right)=2$. Example 3 .

(iv) $f(-1)=0,-1,-2, \ldots$ and $\operatorname{dim}\left(S_{h}\right)=2, S$ is empty. Example 4 .

In all the examples below, the parameters $a, b, c, d, \widetilde{a}, \widetilde{b}, \widetilde{c}, \widetilde{d}, C, \alpha$ and $\beta$ are arbitrary complex numbers. The limits in the $m$ index of the sequences (12) are approximated by the value of the sequences at $m=10$. It has been ckecked that from $m=2$ the ranks of the involved systems get stabilized. We have selected examples for which the general solution of the differential equation is known; in this way we may check the validity of the existence and uniqueness criterion of Section 5 and compare the exact solution(s) with the approximate solution(s) provided by the right hand side of (5) truncated at a certain $n$, that we denote by $p_{n}(z)$. The figures in examples 1,2 and 3 show the relative error in the supremum norm when the exact solution of the corresponding example is approximated by $p_{n}(x), n=0,1, \ldots, 4$, in the interval $x \in(-1,1)$.

Example 1. Consider the boundary value problem:

$$
\left\{\begin{array}{l}
(x+1) y^{\prime \prime}+\frac{3}{2} y^{\prime}-y=C \quad \text { in }(-1,1) \\
a y(-1)+b y(1)+c y^{\prime}(-1)+d y^{\prime}(1)=\alpha \\
\widetilde{a} y(-1)+\widetilde{b} y(1)+\widetilde{c} y^{\prime}(-1)+\widetilde{d} y^{\prime}(1)=\beta
\end{array}\right.
$$

We have $f(x)=3 / 2, g(x)=-1$ and $h(x)=C$. As $f(-1)=3 / 2$, then $n_{0}=0$. For this example, the recurrence relations (8) may be written in the form $v_{n}=M_{n-1} v_{n-1}+c_{n-1}$ with $v_{n}=\left(a_{n}, b_{n}, a_{n+1}, b_{n+1}\right)$, $c_{n}=(0,0,0,0), n=0,1,2, \ldots$, and

$$
M_{n}=\left(\begin{array}{cccc}
0 & 0 & 1 & 0 \\
0 & 0 & 0 & 1 \\
0 & \frac{4 n+7}{8(n+1)(n+2)(2 n+5)} & -\frac{16 n^{2}+48 n+39}{8(n+2)(2 n+5)} & \frac{1}{2(n+2)(2 n+5)} \\
0 & \frac{3}{8(n+1)(n+2)(2 n+5)} & -\frac{12 n+11}{8(n+2)(2 n+5)} & -\frac{2(n+2)}{2 n+5}
\end{array}\right) .
$$

For $m=10$, system (15) is given by

$$
\left\{\begin{array}{l}
-a_{0}+\frac{5 b_{0}}{2}-3 a_{1}+3 b_{1}=C \\
8.08104 b_{0}-45.0381 a_{1}-0.142993 b_{1}=0 \\
3.77407 b_{0}-17.6957 a_{1}-37.4763 b_{1}=0
\end{array}\right.
$$

whose solution is $\left(b_{0}, a_{1}, b_{1}\right)=\left(0.497539 a_{0}+0.497539 C, 0.0892464 a_{0}+0.0892464 C, 0.00796412 a_{0}+\right.$ $0.00796412 C)$, with $a_{0} \in \mathbb{C}$. As $\operatorname{dim}\left(S_{h}\right)=\operatorname{dim}(S)=1$, the differential equation in (22) has a one-dimensional family of analytic solutions in $[-1,1]$. 
Now we apply the existence and uniqueness criterion of Section 5: the existence and uniqueness of solution of (22) is equivalent to the existence and uniqueness of solution of the linear system given by the three equations (23) and the two equations

$$
\left\{\begin{array}{l}
(a+b) a_{0}+(-a+b+c+d) b_{0}+(-2 c+2 d) a_{1}+(2 c+2 d) b_{1}=\alpha \\
(\widetilde{a}+\widetilde{b}) a_{0}+(-\widetilde{a}+\widetilde{b}+\widetilde{c}+\widetilde{d}) b_{0}+(-2 \widetilde{c}+2 \widetilde{d}) a_{1}+(2 \widetilde{c}+2 \widetilde{d}) b_{1}=\beta
\end{array}\right.
$$

Then, problem (22) has a unique solution if and only if

$$
\begin{aligned}
& \frac{\alpha+C(0.497539 a-0.497539 b-0.334974 c-0.69196 d)}{0.502461 a+1.49754 b+0.334974 c+0.69196 d} \\
& =\frac{\beta+C(0.497539 \widetilde{a}-0.497539 \widetilde{b}-0.334974 \widetilde{c}-0.69196 \widetilde{d})}{0.502461 \widetilde{a}+1.49754 \widetilde{b}+0.334974 \widetilde{c}+0.69196 \widetilde{d}} .
\end{aligned}
$$

We next observe that the existence and uniqueness condition obtained with this criterion coincides with the one provided by the knowledge of the family of analytic solutions of the differential equation given in (22):

$$
y\left(x, c_{1}\right):=c_{1} \frac{\sinh (2 \sqrt{x+1})}{\sqrt{x+1}}-C .
$$

The standard criterion of existence and uniqueness of solution of problem (22) depends on the existence of a complex number $c_{1}$ that makes $y\left(x, c_{1}\right)$ compatible with the boundary conditions in $(22)$, that is,

$$
\begin{aligned}
& \frac{\alpha+C(a+b)}{48 a+12 \sqrt{2} \sinh (2 \sqrt{2}) b+32 c+(12 \cosh (2 \sqrt{2})-3 \sqrt{2} \sinh (2 \sqrt{2})) d} \\
& =\frac{\alpha+C(\widetilde{a}+\widetilde{b})}{48 \widetilde{a}+12 \sqrt{2} \sinh (2 \sqrt{2}) \widetilde{b}+32 \widetilde{c}+(12 \cosh (2 \sqrt{2})-3 \sqrt{2} \sinh (2 \sqrt{2})) \widetilde{d}} .
\end{aligned}
$$

It can be checked that (25) and (26) yield to the same condition.

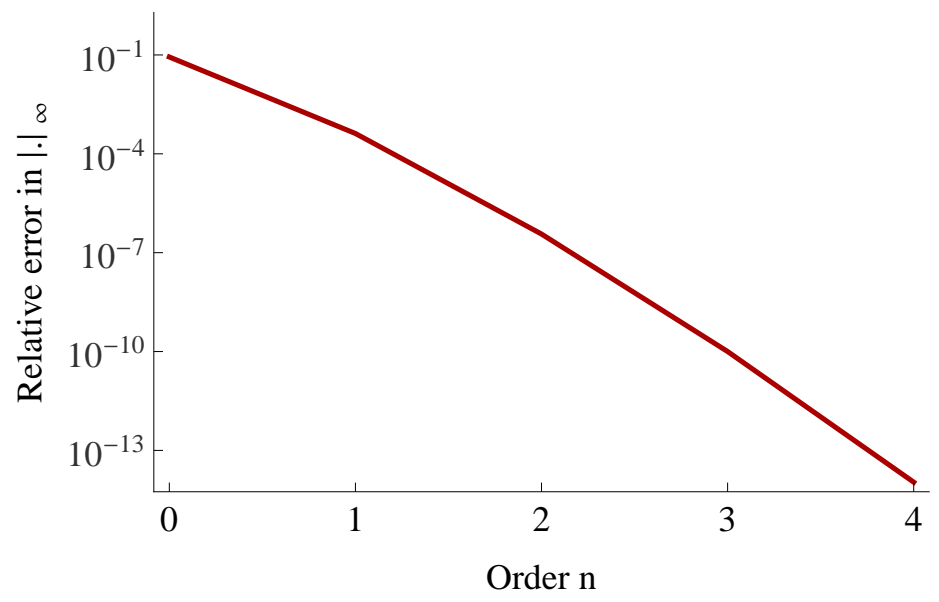

Figure 2: Relative errors in the supremum norm for problem (22) with $C=0, a=b=\widetilde{c}=\widetilde{d}=1, c=d=\widetilde{a}=$ $\widetilde{b}=0, \alpha=1.94755$ and $\beta=1$.

Example 2. Consider the boundary value problem:

$$
\left\{\begin{array}{l}
(x+1) y^{\prime \prime}-y^{\prime}-y=C \quad \text { in }(-1,1) \\
a y(-1)+b y(1)+c y^{\prime}(-1)+d y^{\prime}(1)=\alpha \\
\widetilde{a} y(-1)+\widetilde{b} y(1)+\widetilde{c} y^{\prime}(-1)+\widetilde{d} y^{\prime}(1)=\beta
\end{array}\right.
$$


We have $f(x)=-1, g(x)=-1$ and $h(x)=C$. As $f(-1)=-1$, then $n_{0}=1$. For this example, the recurrence relations (8) may be written in the form $v_{n}=M_{n-1} v_{n-1}+c_{n-1}$ with $v_{n}=$ $\left(a_{n}, b_{n}, a_{n+1}, b_{n+1}, a_{n+2}, b_{n+2}\right), c_{n}=(0,0,0,0,0,0), n=1,2, \ldots$, and

$$
M_{n}=\left(\begin{array}{cccccc}
0 & 0 & 1 & 0 & 0 & 0 \\
0 & 0 & 0 & 1 & 0 & 0 \\
0 & 0 & 0 & 0 & 1 & 0 \\
0 & 0 & 0 & 0 & 0 & 1 \\
0 & 0 & 0 & \frac{2 n+1}{8 n(n+1)(n+2)} & -\frac{4 n^{2}+2 n+1}{4 n(n+2)} & \frac{1}{4 n(n+2)} \\
0 & 0 & 0 & -\frac{1}{8 n(n+1)(n+2)} & \frac{2 n+1}{4 n(n+2)} & -\frac{4 n^{2}+6 n+1}{4 n(n+2)}
\end{array}\right) .
$$

For $m=10$, system (15) is given by

$$
\left\{\begin{array}{l}
-a_{0}+2 a_{1}-2 b_{1}=C, \\
-b_{0}+6 b_{1}+8 a_{2}+8 b_{2}=0 \\
-a_{1}+4 b_{1}+4 a_{2}+4 b_{2}=0 \\
-8.07905 b_{1}+75.7528 a_{2}-11.3031 b_{2}=0 \\
2.78241 b_{1}-29.1304 a_{2}+53.3385 b_{2}=0
\end{array}\right.
$$

whose solution is $\left(b_{0}, a_{1}, b_{1}, a_{2}, b_{2}\right)=\left(a_{0}+C, 0.644633 a_{0}+0.644633 C, 0.144633 a_{0}+0.144633 C, 0.015568 a_{0}+\right.$ $\left.0.015568 C, 0.00095756 a_{0}+0.00095756 C\right)$, with $a_{0} \in \mathbb{C}$. As $\operatorname{dim}\left(S_{h}\right)=\operatorname{dim}(S)=1$, the differential equation in (27) has a one-dimensional family of analytic solutions in $[-1,1]$.

Now we apply the existence and uniqueness criterion of Section 5: the existence and uniqueness of solution of (27) is equivalent to the existence and uniqueness of solution of the linear system given by equations (28) and (24). Then, problem (27) has a unique solution if and only if

$$
\frac{\alpha+C(a-b-2.57853 d)}{2 b+2.57853 d}=\frac{\beta+C(\widetilde{a}-\widetilde{b}-2.57853 \widetilde{d})}{2 \widetilde{b}+2.57853 \widetilde{d}} .
$$

The existence and uniqueness condition obtained with this criterion coincides with the one provided by the knowledge of the family of analytic solutions of the differential equation given in (27):

$$
y\left(x, c_{1}\right)=c_{1}(x+1) I_{2}(2 \sqrt{x+1})-C,
$$

where $I_{\nu}(z)$ is a modified Bessel function. The standard criterion of existence and uniqueness of solution of problem (27) depends on the existence of a complex number $c_{1}$ that makes $y\left(x, c_{1}\right)$ compatible with the boundary conditions in (27), that is,

$$
\begin{aligned}
& \frac{\alpha+C(a+b)}{4 I_{2}(\sqrt{2}) b+\left(\sqrt{2} I_{1}(2 \sqrt{2})+2 I_{2}(2 \sqrt{2})+\sqrt{2} I_{3}(2 \sqrt{2})\right) d} \\
& =\frac{\alpha+C(\widetilde{a}+\widetilde{b})}{4 I_{2}(\sqrt{2}) \widetilde{b}+\left(\sqrt{2} I_{1}(2 \sqrt{2})+2 I_{2}(2 \sqrt{2})+\sqrt{2} I_{3}(2 \sqrt{2})\right) \widetilde{d}} .
\end{aligned}
$$

It can be checked that (29) and (30) are equivalent.

Example 3. Consider the boundary value problem:

$$
\left\{\begin{array}{l}
(x+1) y^{\prime \prime}-\left(1+2 x+2 x^{2}\right) y^{\prime}-2 y=C \quad \text { in }(-1,1), \\
a y(-1)+b y(1)+c y^{\prime}(-1)+d y^{\prime}(1)=\alpha \\
\widetilde{a} y(-1)+\widetilde{b} y(1)+\widetilde{c} y^{\prime}(-1)+\widetilde{d} y^{\prime}(1)=\beta .
\end{array}\right.
$$




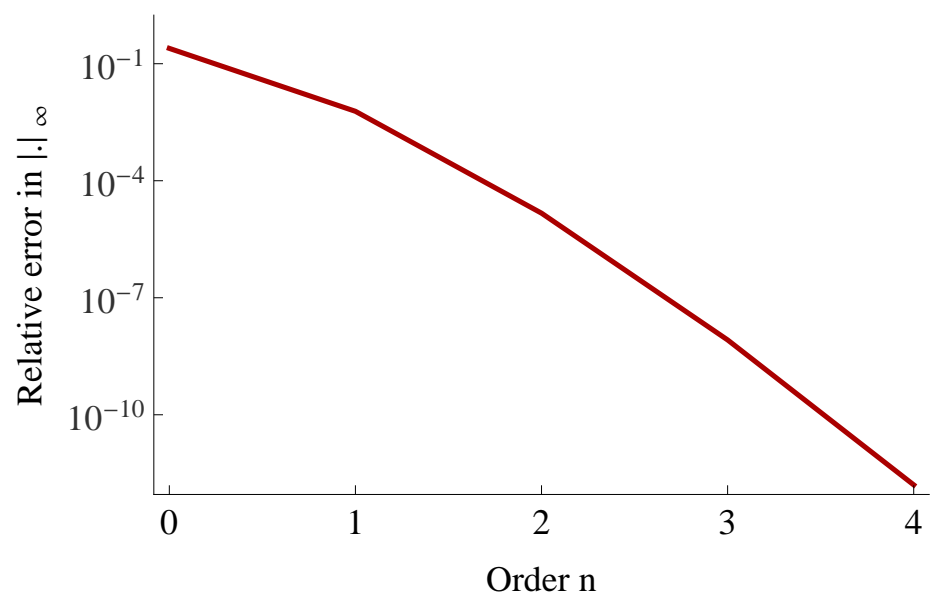

Figure 3: Relative errors in the supremum norm for problem (27) with $C=0, a=b=\widetilde{c}=\widetilde{d}=1, c=d=\widetilde{a}=$ $\widetilde{b}=0, \alpha=0.775635$ and $\beta=1$.

We have $f(x)=-\left(1+2 x+2 x^{2}\right), g(x)=-2$ and $h(x)=C$. As $f(-1)=-1$, then $n_{0}=1$. For this example, the recurrence relations (8) may be written in the form $v_{n}=M_{n-1} v_{n-1}+c_{n-1}$ with $v_{n}=\left(a_{n}, b_{n}, a_{n+1}, b_{n+1}, a_{n+2}, b_{n+2}\right), c_{n}=(0,0,0,0,0,0), n=1,2, \ldots$, and

$$
M_{n}=\left(\begin{array}{cccccc}
0 & 0 & 1 & 0 & 0 & 0 \\
0 & 0 & 0 & 1 & 0 & 0 \\
0 & 0 & 0 & 0 & 1 & 0 \\
0 & 0 & 0 & 0 & 0 & 1 \\
0 & 0 & \frac{2 n+1}{2(n+1)(n+2)} & 0 & -\frac{2 n-1}{2(n+2)} & 0 \\
0 & 0 & -\frac{1}{2(n+1)(n+2)} & \frac{1}{n+2} & \frac{1}{2(n+2)} & -\frac{2 n+1}{2(n+2)}
\end{array}\right) .
$$

For $m=10$, system (15) is given by

$$
\left\{\begin{array}{l}
-2 a_{0}+2 a_{1}+b_{0}-2 b_{1}=C \\
-4 a_{1}+8 a_{2}-4 b_{0}+2 b_{1}+8 b_{2}=0 \\
-2 a_{1}+4 a_{2}-2 b_{0}+b_{1}+4 b_{2}=0 \\
-2.56135 a_{1}+5.1227 a_{2}=0 \\
8.09771 a_{1}-16.1954 a_{2}-14.4782 b_{1}+34.474 b_{2}=0
\end{array}\right.
$$

whose solution is $\left(a_{1}, b_{1}, a_{2}, b_{2}\right)=\left(a_{0}+0.246296 b_{0}+0.5 C, 0.746296 b_{0}, 0.5 a_{0}+0.123148 b_{0}+0.25 C, 0.313426 b_{0}\right)$, with $a_{0}, b_{0} \in \mathbb{C}$. As $\operatorname{dim}\left(S_{h}\right)=\operatorname{dim}(S)=2$, the differential equation in (31) has a two-dimensional family of analytic solutions in $[-1,1]$.

Now we apply the existence and uniqueness criterion of Section 5: the existence and uniqueness of solution of (31) is equivalent to the existence and uniqueness of solution of the linear system given by equations (32) and (24). Then, problem (31) has a unique solution if and only if

$$
\left(\begin{array}{cc}
a+b-2 c+2 d & -a+b+2 c+2.98518 d \\
\widetilde{a}+\widetilde{b}-2 \widetilde{c}+2 \widetilde{d} & -\widetilde{a}+\widetilde{b}+2 \widetilde{c}+2.98518 \widetilde{d}
\end{array}\right)\left(\begin{array}{c}
a_{0} \\
b_{0}
\end{array}\right)=\left(\begin{array}{c}
\alpha+C(c-d) \\
\beta+C(\widetilde{c}-\widetilde{d})
\end{array}\right) .
$$

We next observe that the existence and uniqueness condition obtained with this criterion coincides with the one provided by the knowledge of the general analytic solution of the differential equation given in (31):

$$
y\left(x, c_{1}, c_{2}\right)=c_{1} e^{x^{2}-1}+c_{2}\left(e^{x^{2}} \sqrt{\pi} \operatorname{erf}(x)-1\right)-C / 2 .
$$

The standard criterion of existence and uniqueness of solution of problem (31) depends on the existence of two complex numbers $c_{1}$ and $c_{2}$ that makes $y\left(x, c_{1}, c_{2}\right)$ compatible with the boundary conditions in 
(31), that is,

$$
\left(\begin{array}{ccc}
a+b-2 c+2 d & b(-1+e \sqrt{\pi} \operatorname{erf}(1))+(2 c+2 d-a)(1+e \sqrt{\pi} \operatorname{erf}(1)) \\
\widetilde{a}+\widetilde{b}-2 \widetilde{c}+2 \widetilde{d} & \widetilde{b}(-1+e \sqrt{\pi} \operatorname{erf}(1))+(2 \widetilde{c}+2 \widetilde{d}-\widetilde{a})(1+e \sqrt{\pi} \operatorname{erf}(1))
\end{array}\right)\left(\begin{array}{c}
c_{1} \\
c_{2}
\end{array}\right)=\left(\begin{array}{c}
\alpha+\frac{C(a+b)}{2} \\
\beta+\frac{C(\widetilde{a}+\vec{b})}{2}
\end{array}\right) .
$$

It can be checked that the criterion for the existence and uniqueness of solution of (33) is equivalent to the criterion for the existence and uniqueness of solution of (34).

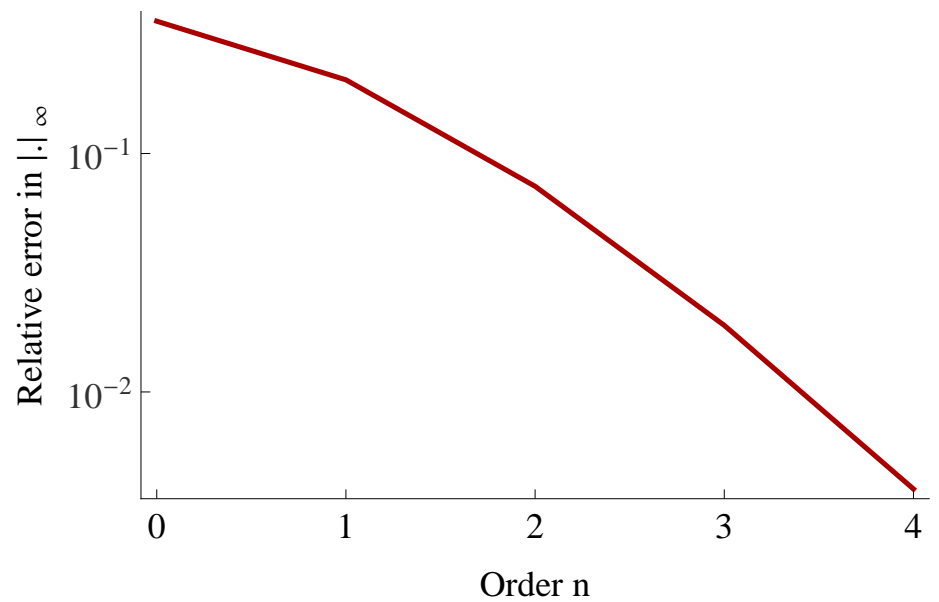

Figure 4: Relative errors in the supremum norm for problem (31) with $C=0, a=b=\widetilde{c}=\widetilde{d}=1, c=d=\widetilde{a}=$ $\widetilde{b}=0, \alpha=\beta=1$.

Example 4. Consider the boundary value problem:

$$
\left\{\begin{array}{l}
(x+1) y^{\prime \prime}=1 \quad \text { in }(-1,1) \\
a y(-1)+b y(1)+c y^{\prime}(-1)+d y^{\prime}(1)=\alpha \\
\widetilde{a} y(-1)+\widetilde{b} y(1)+\widetilde{c} y^{\prime}(-1)+\widetilde{d} y^{\prime}(1)=\beta
\end{array}\right.
$$

We have $f(x)=0, g(x)=0$ and $h(x)=1$. As $f(-1)=0$, then $n_{0}=0$. Then, system (15) consists of 3 equations, the first one is

$$
L_{1}\left[a_{0}, b_{0}, a_{1}, b_{1}\right] \equiv 1=0 .
$$

Thus, problem (35) has no analytic solution in $[-1,1]$. This conclusion is the same one that we obtain from the knowledge of the general solution of the differential equation in (31):

$$
y(x)=c_{1}+c_{2} x+(1+x) \log (1+x) .
$$

\section{Final remarks}

In this paper we have continued and generalized our investigations in the regular case initiated in [8]. The analysis presented in this paper is far more elaborated than the one required in the regular case, as it is necessary a previous study of the spaces $S$ and $S_{h}$ of analytic solutions of the complete and homogeneous equations, and the relation between the size of these spaces and the value of $f(-1)$. Also, the system of recurrences for the two-point Taylor expansion is more complicated and requires an initial seed that depends on the value of $f(-1)$.

In Section 2 we have detailed the dimensionality of the space $S_{h}$ of analytic solutions of the homogeneous equation; the dimension of $S_{h}$ is: (i) one when $f(-1) \neq-1,-2,-3, \ldots$; (ii) one or two 
when $f(-1)=-1,-2,-3, \ldots$; (iii) two when $f(-1)=g(-1)=0$ (regular case). We have included the regular case analyzed in [8] as a particular case of the more general situation analyzed in this paper. The dimension of the space $S$ of analytic solutions of the complete differential equation is either, the same as the dimension of $S_{h}$, or $S$ is empty. A complete characterization of this space is given at the end of Section 4 from the study of the ranks of the algebraic linear systems (15) and (15) $)_{h}$.

In Section 3 we have derived an algorithm to obtain the two-point Taylor expansion of the solutions of (1) (if any). In Section 5 we have given a straightforward and systematic criterion for the existence and uniqueness of analytic solutions of the boundary value problem (1). The criterion is very simple and establishes that the existence and uniqueness of solution of the boundary value problem (1) is equivalent to the existence and uniqueness of solution of the algebraic linear system (21). Two equations of this algebraic system are defined by the limits (12), whose exact computation is, in general, difficult. Then, in practice, the entrances of two of the equations of this algebraic system must be computed approximately and then, the solution is computed in an approximated form. Also, in practice, we must apply the above existence and uniqueness criterion for the solution of (1) using the approximate linear system. Then, the conclusions about the existence and uniqueness of solution are exact unless the system is bad conditioned. In this case, the ranks of the coefficient matrix and/or of the augmented matrix of the system (21) sensibly depend on the precision in the computation of the approximate limits.

Formally, the criterion proposed in this paper is similar to the standard criterion based on the knowledge of the space of solutions: both criteria relate the existence and uniqueness of solution of the boundary value problem (1) to the existence and uniqueness of a solution of an algebraic linear system. As a difference with that standard criterion, our criterion does not require the knowledge of the general solution of the differential equation. This qualitative difference is essential when the general solution of the equation is not known. In this case, the standard criterion is not useful, whereas our criterion can be always applied (except in the case of bad conditioning discussed above), as we have shown in the examples analyzed in Section 7.

\section{Acknowledgments}

The Ministerio de Economía y Competitividad (REF. MTM2014-52859-P) is acknowledged by its financial support.

\section{References}

[1] P. B. Bailey, L. F. Shampine and P. E. Waltman, Nonlinear Two Point Boundary Value Problems, Academic Press, New York, 1968.

[2] C. Kesan, Taylor polynomial solutions of linear differential equations, Appl. Math. Comp., 142 (2003), no. 1, 155165.

[3] A. C. King, J. Billingham and S. R. Отto, Differential Equations. Linear, Nonlinear, Ordinary, Partial, Cambridge Univ. Press, New York, 2003.

[4] P. D. Lax And A. N. Milgram, Parabolic Equations. Contributions to the theory of partial differential equations, Annals of Mathematics Studies. Princeton Univ. Press, 33 (1954), 167-190.

[5] J. L. López, The Liouville-Neumann expansion at a regular singular point, J. Diff. Equ. Appl., 15 (2009), no. 2, 119-132.

[6] J. L. López And Nico M. Temme, Two-point Taylor expansions of analytic functions, Stud. Appl. Math., 109 (2002), no. 4, 297-311.

[7] J. L. López, E. PÉrez Sinusía And N. Temme, Multi-point Taylor approximations in one-dimensional linear boundary value problems, Appl. Math. Comput., 207 (2009), 519-527.

[8] J. L. López AND E. PÉrez Sinusía, Two-point Taylor expansions and one-dimensional boundary value problems, Math. Comput., 79 (2010), no. 272, 2103-2115.

[9] A. B. Olde DaAlhuis and F. W. J. Olver, On the asymptotic and numerical solution of linear ordinary differential equations, SIAM Rev., 40 (1998), no. 3, 463-495. 
[10] F. W. J. Olver, D. W. Lozier, R. F. Boisvert and C. W. Clark, NIST Handbook of Mathematical Functions, Cambridge University Press, Cambridge, 2010. http://dlmf .nist.gov/10.

[11] I. Stakgold, Green's functions and boundary value problems, Wiley and Sons, New York, 1998, Second Edition.

[12] M. SEzER, A method for the approximate solution of the second-order linear differential equations in terms of Taylor polynomials, Int. J. Math. Edu. Sci. Technol., 27 (1996), 821-834.

[13] I. Stakgold, Green's functions and boundary value problems, Wiley and Sons, New York, 1998. 